\title{
Fully Distributed Consensus of Fractional Chaotic Multi-agent Systems Based on Combined Event-triggered Mechanism
}

\author{
Qiaoping $\mathrm{Li}^{1}$ and Chao Yue ${ }^{1}$ \\ ${ }^{1}$ Zhengzhou University of Aeronautics
}

June 12, 2021

\begin{abstract}
By designing a novel combined event-triggered control technique, this article analytically studies the distributed leader-following consensus problem of nonlinear fractional chaotic multi-agent systems. First, a novel combined event-triggered mechanism which takes into account both the relative error and the absolute error of the samples is proposed, under which each follower agent executes control update independently at its own event times. Next, a fully distributed event-triggered consensus protocol is designed and the sufficient conditions of consensus are attained. Finally, compared with other event-triggered mechanisms, the simulation experiments illustrate that the event-based consensus protocol proposed in this article can effectively reduce the frequency of actuator data update while ensuring desired consensus performance.
\end{abstract}

\section{Hosted file}

T10_13.pdf available at https://authorea.com/users/419488/articles/525972-fully-distributedconsensus-of-fractional-chaotic-multi-agent-systems-based-on-combined-event-triggeredmechanism

\section{Hosted file}

T10_13.tex available at https://authorea.com/users/419488/articles/525972-fully-distributedconsensus-of-fractional-chaotic-multi-agent-systems-based-on-combined-event-triggeredmechanism

\section{Hosted file}

CETM_10_3.eps available at https://authorea.com/users/419488/articles/525972-fullydistributed-consensus-of-fractional-chaotic-multi-agent-systems-based-on-combined-eventtriggered-mechanism 
figures $/ \mathrm{x} 0 / \mathrm{x} 0$-eps-converted-to.pdf 
figures/ee-c-123456/ee-c-123456-eps-converted-to.pdf 
figures/event-time-c/event-time-c-eps-converted-to.pdf 
figures/ee-n-123456/ee-n-123456-eps-converted-to.pdf 
figures/event-time-n/event-time-n-eps-converted-to.pdf 
figures/ee-e-123456/ee-e-123456-eps-converted-to.pdf 
figures/event-time-e/event-time-e-eps-converted-to.pdf 
figures/number-N-E-C/number-N-E-C-eps-converted-to.pdf 
figures/ee-c-unified-123456/ee-c-unified-123456-eps-converted-to.pdf 
figures/event-time-c-unifeid/event-time-c-unifeid-eps-converted-to.pdf 
figures/updatenumber-unifedC/updatenumber-unifedC-eps-converted-to.pdf 